\title{
Experimental Infection of Cynomolgus Macaques (Macaca fascicularis) with Aerosolized Monkeypox Virus
}

\author{
Aysegul Nalca ${ }^{1}$, Virginia A. Livingston ${ }^{1}$, Nicole L. Garza ${ }^{1}$, Elizabeth E. Zumbrun ${ }^{1}$, Ondraya M. Frick ${ }^{1}$, \\ Jennifer L. Chapman ${ }^{2}$, Justin M. Hartings ${ }^{1 \times}$
}

1 Center for Aerobiological Sciences, United States Army Medical Research Institute of Infectious Diseases (USAMRIID), Fort Detrick, Maryland, United States of America, 2 Pathology Division, United States Army Medical Research Institute of Infectious Diseases (USAMRIID), Fort Detrick, Maryland, United States of America

\begin{abstract}
Monkeypox virus (MPXV) infection in humans results in clinical symptoms very similar to ordinary smallpox. Aerosol is a route of secondary transmission for monkeypox, and a primary route of smallpox transmission in humans. Therefore, an animal model for aerosol exposure to MPXV is needed to test medical countermeasures. To characterize the pathogenesis in cynomolgus macaques (Macaca fascicularis), groups of macaques were exposed to four different doses of aerosolized MPXV. Blood was collected the day before, and every other day after exposure and assessed for complete blood count (CBC), clinical chemistry analysis, and quantitative PCR. Macaques showed mild anorexia, depression, and fever on day 6 post-exposure. Lymphadenopathy, which differentiates monkeypox from smallpox, was observed in exposed macaques around day 6 post-exposure. CBC and clinical chemistries showed abnormalities similar to human monkeypox cases. Whole blood and throat swab viral loads peaked around day 10, and in survivors, gradually decreased until day 28 post-exposure. Survival was not dose dependent. As such, doses of $4 \times 10^{4} \mathrm{PFU}, 1 \times 10^{5} \mathrm{PFU}$, or $1 \times 10^{6} \mathrm{PFU}$ resulted in lethality for $70 \%$ of the animals, whereas a dose of $4 \times 10^{5}$ PFU resulted in $85 \%$ lethality. Overall, cynomolgus macaques exposed to aerosolized MPXV develop a clinical disease that resembles that of human monkeypox. These findings provide a strong foundation for the use of aerosolized MPXV exposure of cynomolgus macaques as an animal model to test medical countermeasures against orthopoxviruses.
\end{abstract}

Citation: Nalca A, Livingston VA, Garza NL, Zumbrun EE, Frick OM, et al. (2010) Experimental Infection of Cynomolgus Macaques (Macaca fascicularis) with Aerosolized Monkeypox Virus. PLoS ONE 5(9): e12880. doi:10.1371/journal.pone.0012880

Editor: Patricia V. Aguilar, Naval Medical Research Center Detachment, Peru

Received May 24, 2010; Accepted August 11, 2010; Published September 20, 2010

This is an open-access article distributed under the terms of the Creative Commons Public Domain declaration which stipulates that, once placed in the public domain, this work may be freely reproduced, distributed, transmitted, modified, built upon, or otherwise used by anyone for any lawful purpose.

Funding: This study was supported by an interagency agreement (IAA: A120-B11) between The Office of Biodefense Research Affairs (OBRA)/National Institute of Allergy and Infectious Diseases (NIAID) and USAMRIID. The sponsors had input in study design and review of the manuscript, but had no role in data collection and analysis, decision to publish, or preparation of the manuscript.

Competing Interests: The authors have declared that no competing interests exist.

*E-mail: aysegul.nalca@us.army.mil

a Current address: Biaera Technologies, LLC, Hagerstown, Maryland, United States of America

\section{Introduction}

After a centuries-long battle, humans defeated smallpox and it was declared eradicated in 1980. Smallpox vaccination was then discontinued, leaving most of today's population vulnerable to Variola virus (VARV) (smallpox agent). MPXV is a close relative of VARV, sharing $96.3 \%$ identity within the central region of the genome encoding essential genes, and $84.5 \%$ identity overall [1]. MPXV causes a disease in humans that is clinically indistinguishable from ordinary smallpox, with the exception of lymphadenopathy $[1,2,3]$. Vaccinia-based vaccines, used for worldwide eradication of VARV, are protective against MPXV challenge in animal models and are also presumed to protect humans from monkeypox $[4,5,6,7,8,9,10,11,12]$. The similarity of monkeypox to smallpox, and the growing lack of immunity in the population have caused concerns that these viruses might be used as biological weapons. This has prompted scientists to develop new medical countermeasures against poxviruses. Because evaluation of medical countermeasures against poxvirus infection in humans is not ethical or feasible, showing efficacy in an animal model that emulates human disease is required by the "Animal Rule" of the US Food and Drug and Administration (FDA) [13]. Non-human primates (NHPs) are closely related to humans and are often the most accurate model system for the study of human disease processes. Therefore it is important to develop a model of MPXV infection in NHPs, using the most relevant route, in order to fully evaluate pathogenesis as well as the capabilities of vaccines and therapeutics.

The use of VARV in research is highly restricted; therefore, viruses from other members of the orthopoxvirus family are used to develop animal models to test medical countermeasures against poxviruses. There are few orthopoxvirus animal disease models that simulate the pathophysiology and unique clinical progression of smallpox and monkeypox in humans. The current models utilize a wide range of orthopoxviruses, animal species, and challenge routes. The current animal models include: vaccinia virus in mice by intranasal (i.n.), intraperitoneal (i.p.) or intravenous (i.v.) routes, cowpox virus in mice and marmosets by the i.n. route, ectromelia in mice by aerosol and i.n. routes, vaccinia virus or rabbitpox virus in rabbits by intradermal (i.d.) or aerosol routes, monkeypox virus in dormice, prairie dogs or ground squirrels by i.n. or i.p. routes, monkeypox virus in monkeys by intratracheal (i.t.) and i.v. routes, and VARV in monkeys by the i.v. route $+/-$ aerosol route $[12,14,15,16,17,18,19,20,21]$. 
It is well established that VARV is transmitted by the aerosol route [22]. Furthermore, an intentional release of VARV or MPXV would likely be in aerosol form. Therefore, the aerosol route of transmission should be one of the features of an animal model which will be used as a model for human smallpox and monkeypox infection. No single model recapitulates all the aspects of smallpox or monkeypox in humans, yet the most relevant models, MPXV or VARV infection of NHPs by the aerosol route, have not been fully characterized $[23,24,25]$. Studies of aerosol MPXV infection models require biosafety level 3 (BSL-3) laboratories and class III biosafety cabinets containing specialized aerosol equipment. The single published study of aerosolized MPXV infection of cynomolgus macaques gave a detailed account of the pathology induced by the virus in various tissues, but did not address a number of facets of the clinical disease progression $[23,25]$. We present that cynomolgus macaques exposed to aerosolized MPXV show many characteristics of monkeypox and smallpox in humans and is thus an appropriate model for orthopoxvirus pathogenesis, vaccine and therapeutic studies.

\section{Materials and Methods}

\section{Animals and Ethical Statement}

Healthy, adult cynomolgus macaques (Macaca fascicularis) of both sexes were obtained from the United States Army Medical Research Institute of Infectious Diseases (USAMRIID) NHP colony. All MPXV exposed animals were handled in a BSL-3 containment laboratory at USAMRIID. Research was conducted in compliance with the Animal Welfare Act and other federal statutes and regulations relating to animals and experiments involving animals, and adhered principles stated in the Guide for the Care and Use of Laboratory Animals, National Research Council, 1996. The facility where this research was conducted (USAMRIID) is fully accredited by the Association for the Assessment and Accreditation of Laboratory Animal Care International. Research was conducted under a protocol approved by the Institutional Animal Care and Use Committee (IACUG) at USAMRIID. All animals were examined and evaluated twice per day by study personnel. Early endpoint criteria, as specified by the score parameters within the "Post-exposure observations" section of these methods, were used to determine when animals should be humanely euthanized.

\section{Virus}

MPXV (Zaire V79-I-005 strain) was provided by the Biodefense and Emerging Infections Research Resources Repository (BEI Resources)/ATCG. Virus was diluted in Eagle's Minimum Essential Medium (EMEM) with 2\% fetal bovine serum (FBS) to achieve desired doses (Table 1).

\section{Plaque Assay}

MPXV was titrated in complete Eagles Minimum Essential Media containing non-essential amino acids (EMEM/NEAA media) supplemented with $2 \% \mathrm{FBS}$, penicillin $(8 \mathrm{IU} / \mathrm{mL})$, streptomycin $(80 \mu \mathrm{g} / \mathrm{mL})$, gentamicin $(0.02 \mathrm{mg} / \mathrm{mL})$ and fungizone $(0.1 \mathrm{U} / \mathrm{mL})$ warmed to $37^{\circ} \mathrm{C}$. Plaque assays were carried out on Vero E6 cells at approximately 95\% confluency. One hundred $\mu \mathrm{L}$ of each dilution was added to each well of a sixwell plate. Plates were incubated for 1 hour in at $37^{\circ} \mathrm{C}$, rocking plates every 10-15 minutes. After one hour, two $\mathrm{mL}$ of complete media were added to each well and incubated for 4 days at $37^{\circ} \mathrm{C}$. On day 4 , media was removed from the plates and $500 \mu \mathrm{L} 10 \%$ crystal violet was added to each well for approximately 10-20 minutes. Once cells were stained, plates were rinsed by submersion in cold water and placed upside down to dry overnight. Plaques were counted the following day.

\section{Aerosol exposures}

Each macaque was anesthetized by intramuscular (i.m.) injection of tiletamine/zolazepam $(6 \mathrm{mg} / \mathrm{kg})$, and whole body plethysmography (Buxco Research Systems, Wilmington, NG) was performed to determine the respiratory minute volume as previously described [26]. Subsequently, each macaque was exposed to MPXV in a head-only chamber contained in a class III biological safety cabinet located inside a BSL-3 suite. The Automated Bioaerosol Exposure System (ABES) served as the control platform for the aerosol exposures [27]. Aerosols were generated with a three-jet collison nebulizer (BGI, Inc., Waltham, MA), and integrated air samples were collected throughout the exposure with an all glass impinger (AGI).

To improve the precision of presented aerosol doses, the ABES was programmed to dynamically calculate the exposure time based on the minute volume measurement for each macaque, the flow to volume ratio of the exposure chamber, the starting MPXV concentration in the collison nebulizer, and the historical spray factor for MPXV virus. For each individual exposure, these parameters were entered into the ABES, and the ABES determined the time required to reach the required dose. ABES calculations were based on a dynamic model that estimates chamber aerosol concentration based on the flow to volume ratio in the chamber.

After exposure, AGI samples were analyzed by performing a plaque assay. An inhaled MPXV dose was calculated for each macaque based on the plaque assay and the minute volume measurement.

\section{Telemetry}

A radiotelemetry device (Data Sciences International (DSI), St. Paul, MN), used to monitor temperature and activity, was surgically implanted into macaques at least 14 days before aerosol exposure. Body temperatures were recorded every $15 \mathrm{~min}$ by the DataQuest A.R.T.4.1 system (DSI). Pre-exposure temperature data were used to create a baseline to fit an autoregressive integrated moving average (ARIMA) model. Temperature elevations exceeding three standard deviations over the baseline were used to compute fever duration, hours, and average elevation.

\section{Post-exposure observations}

Macaques were observed at least twice a day after aerosol exposure. Macaques were scored for clinical signs of disease prior to, and while under anesthesia. The scoring parameters were: responsiveness and appearance (0: active; 2: depression, mild unresponsiveness; 3: head down, hunched; 4: moderate unresponsiveness; 5: severe unresponsiveness), dyspnea (0: normal breathing; 2: mildly labored; 3: labored; 5: agonal breathing), dehydration (0: not present; 1: mild; 2: moderate; 3: severe), anorexia (0: eating; 1: no biscuits for 1 day but eats enrichment; 2: no biscuits for 2 days or not eating enrichment), rash (0: none; 1 : slight; 2: moderate; 3 : severe), cough (0: none; $1: \leq 2$ coughs/ $5 \mathrm{~min}$; 2: 3-10 coughs/5 $\mathrm{min} ; 3: \geq 10$ coughs/5 min), nasal discharge (0: none; 1: mild; 2: moderate; 3 : severe), urine $(0$ : normal; 3: none), stool (0: normal; 1: loose stool, 2: liquid stool or none), and fever ( 0 : no change, 1 : baseline $+1^{\circ} \mathrm{C}$; 2 : baseline $+2^{\circ} \mathrm{C}$ or higher; 3: baseline $-2^{\circ} \mathrm{C}$ ). Macaques were also evaluated for changes in weight (0: no change; 1 : baseline-10-15\%; 2: baseline $15 \%$; 3 : baseline $-20 \%)$ and the presence of lymphadenopathy $(0$ : $<3 \mathrm{~mm}$; 1: 3-9 mm; 2: >10-19 mm; 3: >20 mm). The early endpoint criteria for humane euthanasia, indicative of very poor 
Table 1. Summary of inhaled doses, fever, and disease outcome in cynomolgus macaques exposed to aerosolized MPXV.

\begin{tabular}{|c|c|c|c|c|c|c|c|c|}
\hline Groups (PFU) & $\begin{array}{l}\text { Inhaled dose } \\
\text { (PFU) (average) }\end{array}$ & $\begin{array}{l}\text { Fever onset } \\
\text { (study day) }^{\mathrm{a}}\end{array}$ & $\begin{array}{l}\text { Fever duration } \\
\text { (hours) }\end{array}$ & Fever hours $^{c}$ & $\Delta \mathrm{T}_{\max }{ }^{\circ} \mathrm{C}^{\mathrm{d}}$ & $\begin{array}{l}\text { Average elevation } \\
\text { in temperature } \\
\left({ }^{\circ} \mathrm{C}\right)^{\mathrm{e}}\end{array}$ & MTD (days) ${ }^{f}$ & $\begin{array}{l}\text { Survivors/ } \\
\text { total }\end{array}$ \\
\hline $4 \times 10^{4}$ & $4.3 \times 10^{4}$ & 4.7 & 105.3 & 215.3 & 2.5 & 1.9 & 10.0 & $1 / 3$ \\
\hline $1 \times 10^{5}$ & $1.4 \times 10^{5}$ & 3.8 & 121.5 & 244.7 & 3.3 & 1.9 & 9.0 & $2 / 6$ \\
\hline $4 \times 10^{5}$ & $4.4 \times 10^{5}$ & 2.8 & 120.8 & 266.7 & 3.4 & 2.1 & 9.6 & $1 / 6$ \\
\hline $1 \times 10^{6}$ & $1.1 \times 10^{6}$ & 4.3 & 123.6 & 278.1 & 3.5 & 2.3 & 8.5 & $1 / 3$ \\
\hline
\end{tabular}

${ }^{\mathrm{a}}$ Defined as the first day with $>8 \mathrm{~h}$ of significant temperature elevation (as determined by ARIMA modeling).

${ }^{b}$ Calculated as the number of days (converted to hours) with 12 or more $\mathrm{h}$ of significant temperature elevation.

cCalculated as the sum of the significant temperature elevations.

${ }^{\mathrm{d}}$ The maximum change in temperature.

${ }^{\mathrm{e}}$ Calculated by dividing fever hours by fever duration in hours.

fMean time-to-death.

doi:10.1371/journal.pone.0012880.t001

health status, were cumulative clinical scores of 15-20 (maximum score), and/or a sudden drop of $>3{ }^{\circ} \mathrm{C}$ from baseline body temperature.

\section{Clinical laboratory evaluations}

Beginning one day before, and every other day on days 2-28 after exposure, blood samples were collected from the femoral vein of macaques anesthetized with tiletamine/zolazepam $(3 \mathrm{mg} / \mathrm{kg}$; IM). Samples collected one day prior to exposure served as a normal reference baseline for each animal. CBCs and blood chemistries were analyzed with Beckman Coulter hematology and VITROS 250 chemistry analyzers.

\section{DNA extraction and real-time polymerase chain reaction (RT-PCR)}

Tissue samples were collected from all major organ systems and frozen. One gram of each tissue was pulverized with $1 \mathrm{~mL}$ EMEM plus $2.5 \mathrm{~mL}$ penicillin $(20,000 \mathrm{IU} / \mathrm{mL}) /$ streptomycin $(200,000$ $\mu \mathrm{g} / \mathrm{mL}$ ) solution using a handheld Omni International (Kennesaw, GA) tissue homogenizer with a single use disposable plastic tissue grinding tip. Blood and throat swabs were collected from macaques every other day after exposure to aerosolized MPXV. Viral DNA was isolated from $200 \mu \mathrm{l}$ of tissue suspension, $100 \mu \mathrm{l}$ of blood, or $100 \mu \mathrm{l}$ of throat swab media with a BioRobot M48 (Qiagen, Valencia, CA) according to the manufacturer's instructions. Real-time PCR was performed with the LightCycler (Roche, Indianapolis, IN) using a pan-orthopoxvirus hemagglutinin assay (HA), as previously described [28].

\section{Necropsy}

A necropsy was performed under BSL-3 conditions on animals that were humanely euthanized when moribund or, if they survived, at the end of the study. Tissue samples from all major organ systems (respiratory, gastrointestinal, genitourinary, lymphoid, neurologic, endocrine, skin and mucous membranes) were collected from each animal for histopathological and immunohistochemical examination and were immersion-fixed in $10 \%$ neutral-buffered formalin.

\section{Histology and immunohistochemistry}

Formalin-fixed tissues for histologic examination were trimmed, processed, and embedded in paraffin according to established protocols [29]. Histology sections were cut at $5 \mu \mathrm{m}$, mounted on glass slides, and stained with hematoxylin and eosin $(\mathrm{H} \& \mathrm{E})$. Immunohistochemical staining was performed on replicate tissues sections using an EnVision + kit (DAKO, Carpinteria, CA). Normal splenic tissue served as the negative control; the positive control was spleen from a known MPXV infected NHP; and normal rabbit serum was used as the negative serum control.

Briefly, sections were deparaffinized in xyless, rehydrated in graded ethanol, and endogenous peroxidase activity was quenched in a $0.3 \%$ hydrogen peroxide/methanol solution for $30 \mathrm{~min}$ at room temperature. Slides were washed in phosphate buffered saline (PBS) then sections were incubated in the primary antibody, a rabbit polyclonal antibody against vaccinia virus, diluted 1:3500 for 60 minutes at room temperature. Sections were washed in PBS and incubated for $30 \mathrm{~min}$ with EnVision + rabbit secondary reagent (horseradish peroxidase-labeled polymer) at room temperature. Peroxidase activity was developed with 3, 3'-diaminobenzidine $(\mathrm{DAB})$, counterstained with hematoxylin, dehydrated, cleared with xyless, then coverslipped.

\section{Digital Microscopy Image Analysis}

Digital microscopy was performed using an Automated Cellular Imaging System $\left(\right.$ ACIS $^{\circledR}$ II, Dako, Carpenteria, CA) which uses proprietary software to allow for color detection and analysis of morphometric features. This system consists of an automated robotic bright-field microscope module, a computer, and a Microsoft Windows NT based software interface. The robotic microscope module scanned the immunohistochemically stained slides and the digitized images were displayed on the computer monitor. The pathologist reviewed the images and an ACISassisted score was generated by the system software as a means of quantitating the vaccinia virus staining in the tissue. Technical details about the ACIS digital microscopy system are presented elsewhere [30].

\section{Statistical analysis}

Repeated measures analysis of variance (RM-ANOVA) was used to compare temperature, weight, white blood cells (WBC), blood chemistries, and viral genomes among groups until day 10 , since after that time, the $1 \times 10^{5}$ PFU group had two survivors and the other groups had one survivor each. Group comparisons for time-to-death were calculated by t-tests with step-down Bonferrini correction. RM-ANOVA of $\log _{10}$ transformed data was used for comparison of viral genome load in blood and throat swabs between groups over time. T-tests of $\log _{10}$ transformed data were performed for viral genome load from tissues between groups. Analyses were two-tailed and conducted using SAS v9.1.3. Results were significant at $P<0.05$. 


\section{Results}

\section{Clinical signs and survival}

In this study, four groups of cynomolgus macaques were exposed to increasing doses of aerosolized MPXV, with a particle size of $1-3 \mu \mathrm{m}$ (Table 1). As little as 200 pfu MPXV delivered by the aerosol route was sufficient to cause non-lethal disease, including lesions (data not shown). The doses were $4 \times 10^{4} \mathrm{PFU}$ $(\mathrm{n}=3), 1 \times 10^{5} \mathrm{PFU}(\mathrm{n}=6), 4 \times 10^{5} \mathrm{PFU}(\mathrm{n}=6)$, and $1 \times 10^{6} \mathrm{PFU}$ $(n=3)$, chosen based on optimization of the dose range required for consistent infection (data not shown). The calculated inhaled doses were very close to target doses for each group (Table 1).

Animals started to show clinical signs of disease, including decreased appetite and activity, by day 3. Lymphadenopathy of inguinal and axillary nodes was observed starting 6-7 days postexposure. By 6-8 days post-exposure, macules began to form in all animals and macaques were also inactive, somnolent, and exhibited depressed posture. The clinical score of all macaques peaked on day 10 post-exposure and there were no significant differences among groups (Figure 1A). Lesions progressed to papules by day 10 and evolved to vesicular and pustular stages by 12-14 days post-exposure. Surviving macaques were active, eating well, had scabbed lesions and had greatly reduced clinical scores by day 20 . Of note, the number of lesions was not dose dependent and varied widely with 60-730 lesions among survivors, and 10-
180 lesions among 11 of 13 non-survivors. Two non-survivors had too many lesions to count $(>2000)$.

Survival was not dose dependent, with doses of $4 \times 10^{4} \mathrm{PFU}$, $1 \times 10^{5} \mathrm{PFU}$, and $1 \times 10^{6} \mathrm{PFU}$ resulting in $33 \%$ survival and $4 \times 10^{5}$ PFU resulting in approximately $17 \%$ survival (Fig. 1B). Although death was delayed in the lowest dose group, there were no significant differences in the mean time-to-death (MTD) (Table 1). Most of the macaques met criteria for euthanasia on days 8-11. Surviving macaques had less severe disease, except for one macaque in the highest dose group, which had severe disease but never met the score criteria for euthanasia.

\section{Body temperature and weight changes}

Fever was delayed until day 5 in the lowest dose group compared to day 4 for the other groups (Table 1 and Figure 1G). However, differences were not significant between the groups regarding onset, duration, or magnitude of fever. By day 5 post-exposure, all groups had an average elevation in body temperature of $2^{\circ} \mathrm{C}$. The lowest dose group had the shortest fever duration, whereas the highest dose group had the longest. Similarly, the lowest dose group had the fewest fever hours, calculated as the sum of significant temperature elevations, and the highest dose group had the most.

Even though there were no significant weight changes over time among groups after MPXV exposure, weight was significantly different between survivors and non-survivors regardless of dose
A

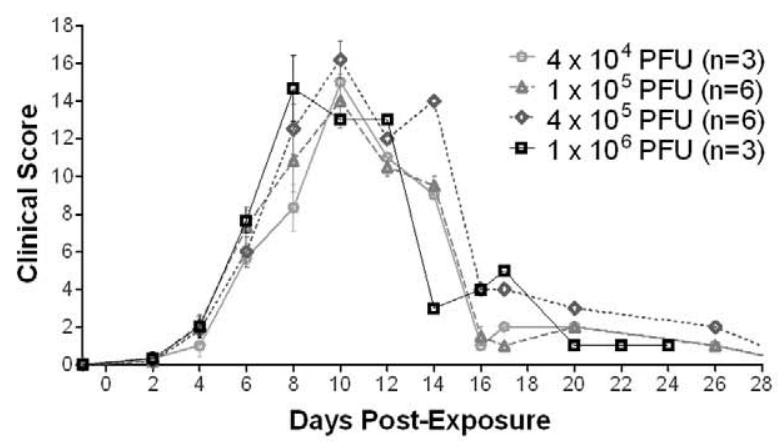

C

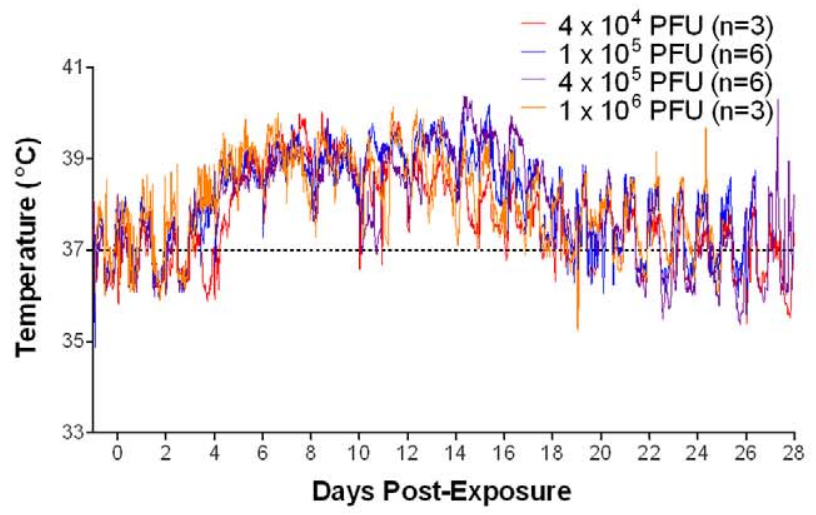

B

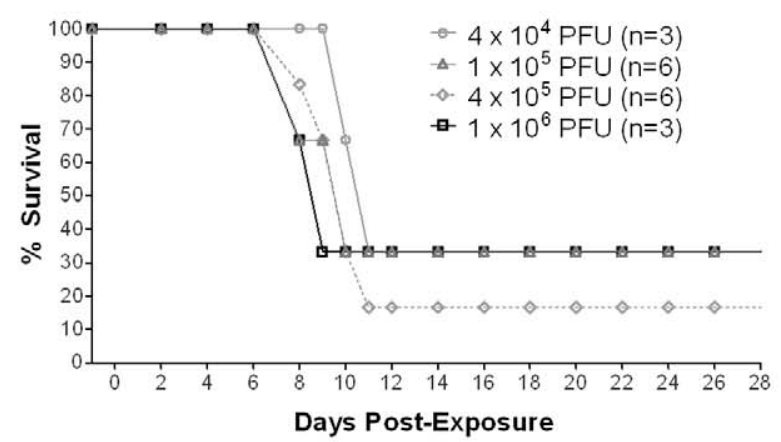

D

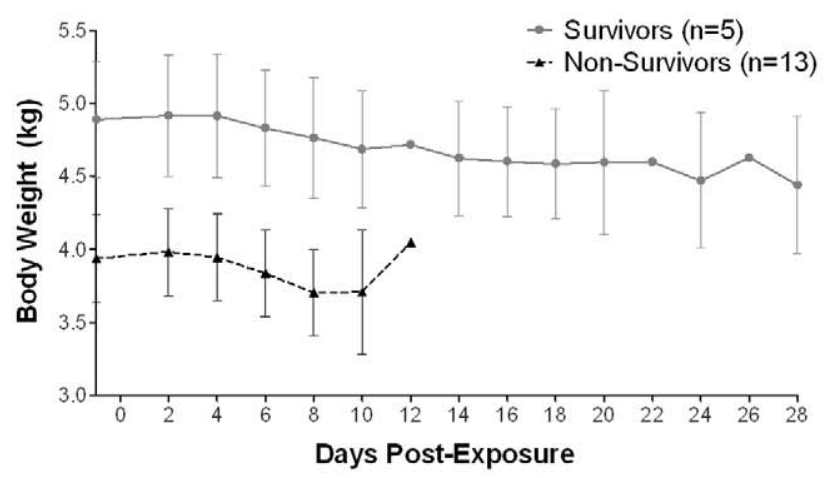

Figure 1. Clinical disease scores, survival, temperature and weight. A) Changes in average clinical scores and B) percent survival of macaques exposed to different doses of aerosolized MPXV. C) Average body temperature of macaques exposed to different doses of aerosolized MPXV. The temperature data was collected every $15 \mathrm{~min}$ from implanted telemetry devices. The dashed line indicates the average baseline body temperature; $n$ : number of animals. D) Changes in average body weight of survivors and non-survivors exposed to aerosolized MPXV. n: number of animals.

doi:10.1371/journal.pone.0012880.g001 
$(P<0.0001)$ (Figure 1D). Survivors were approximately $20 \%$ heavier than non-survivors.

Clinical laboratory evaluation

In contrast to a previous report on aerosol infection of cynomolgus macaques with MPXV which reported no significant changes in CBCs or blood chemistries after infection, a number of changes were observed in this study [25]. WBCs decreased slightly on day 2 and increased after day 4 in all groups except for the lowest dose group $(P=0.0011)$ (Fig. 2A). All three dosage groups had data points that were above the normal reference range on certain days. Granulocytes decreased on day 2, followed by a
A

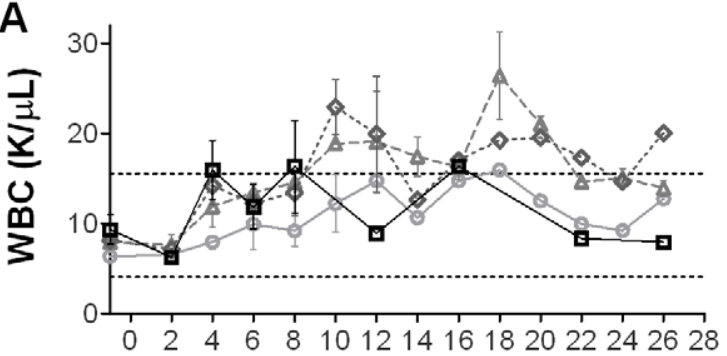

B

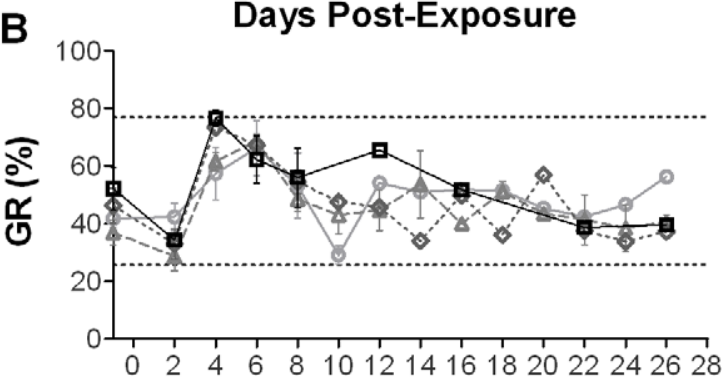

Days Post-Exposure

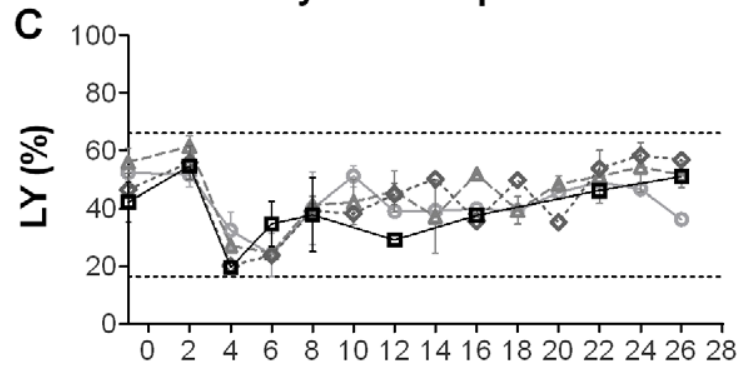

Days Post-Exposure

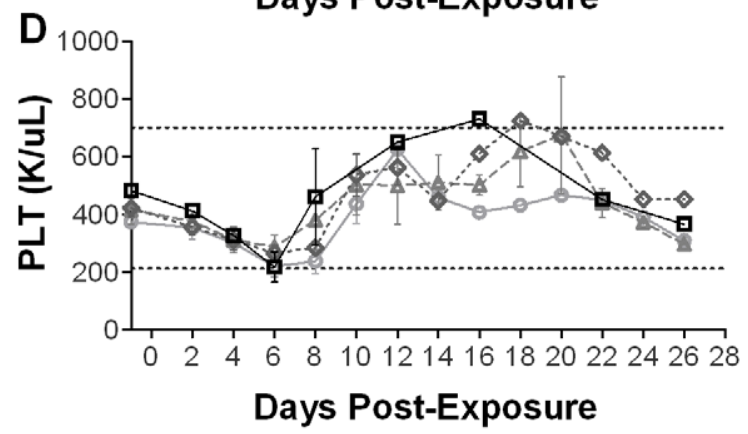

$\begin{array}{ll}- & 4 \times 10^{4} \text { PFU }(n=3) \\ -\triangle & 1 \times 10^{5} \text { PFU }(n=6) \\ -\diamond & 4 \times 10^{5} \text { PFU }(n=6) \\ \text { - } & 1 \times 10^{6} \text { PFU }(n=3)\end{array}$

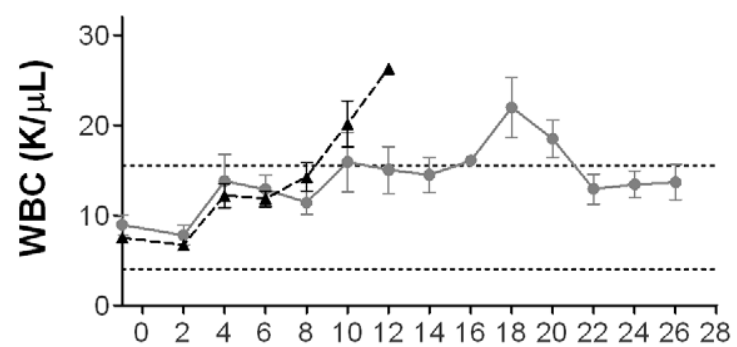

Days Post-Exposure
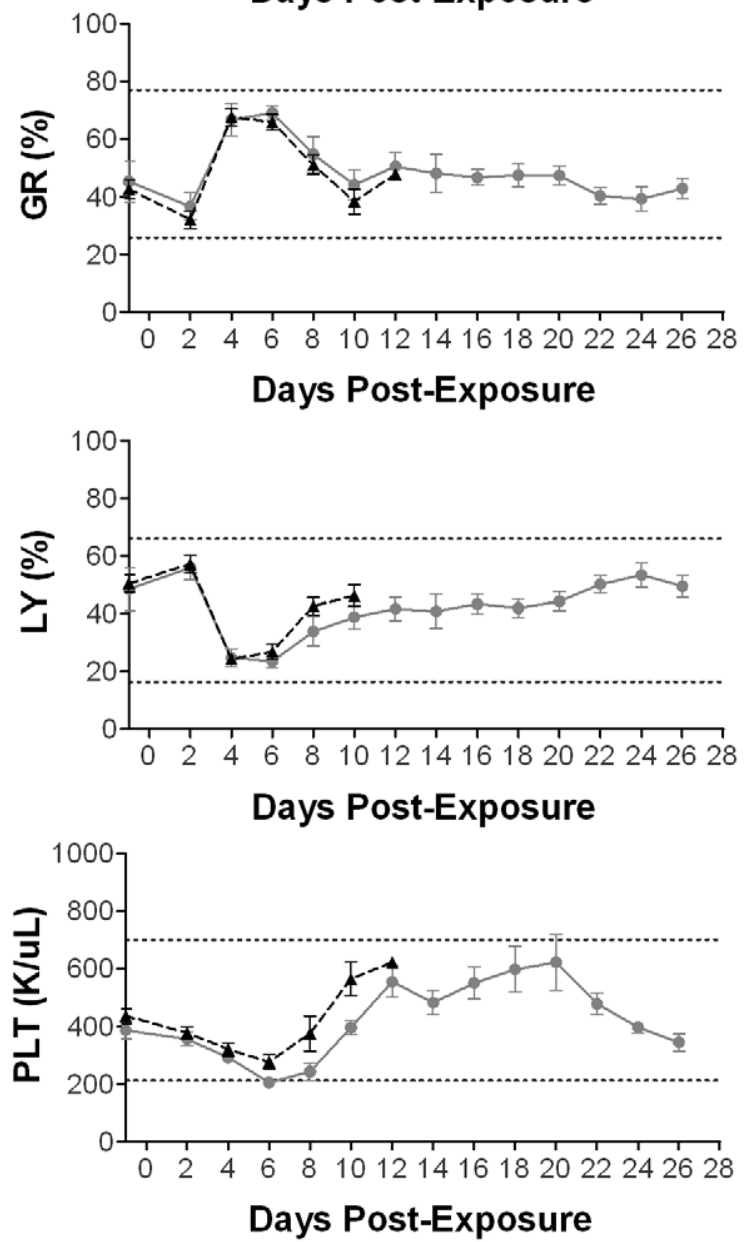

$\rightarrow$ Survivors $(n=5)$

-4. Non-Surivors $(n=13)$

Figure 2. Average number of leukocytes and platelets in macaques after exposed to aerosolized MPXV. The dotted lines indicate the normal reference range; $n$ : number of animals. Graphs are shown for A) total white blood cells (WBC), B) percentage of granulocytes (GR), C) percentage of lymphocytes (LY), D) platelets (PLT) for all MPXV dosage groups, and survivors versus non-survivors (right).

doi:10.1371/journal.pone.0012880.g002 
sharp increase on day 4 post-exposure (Fig. 2B). In contrast, peripheral lymphocytes increased on day 2 followed by a sharp decrease on day 4 post-exposure (Fig. 2C). There were no significant differences on days $-1-10$ between survivors and nonsurvivors regarding changes in WBC, granulocyte, or lymphocyte values.
Platelets decreased steadily from day 2 to day 6 . Platelets were lowest on day 6 post-exposure, which was significantly lower than day $-1(P<0.0001)$, and increased after day 6 but stayed within the normal range (Fig. 2D). Interestingly, non-survivors had significantly higher levels of platelets than survivors on days -1 to $10(P=0.0011)$.
A

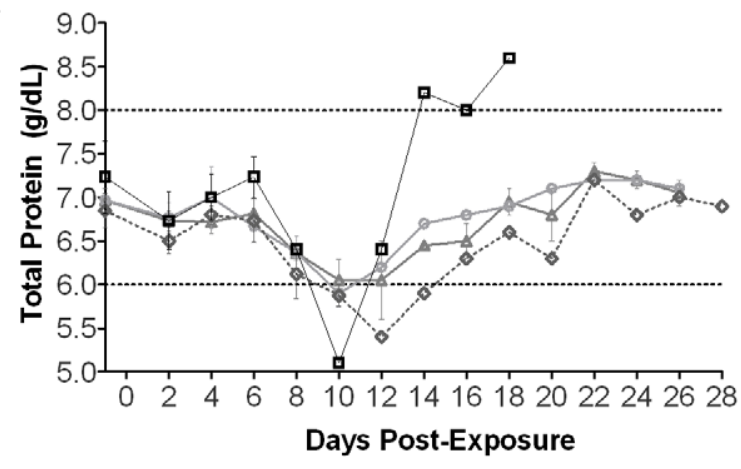

C

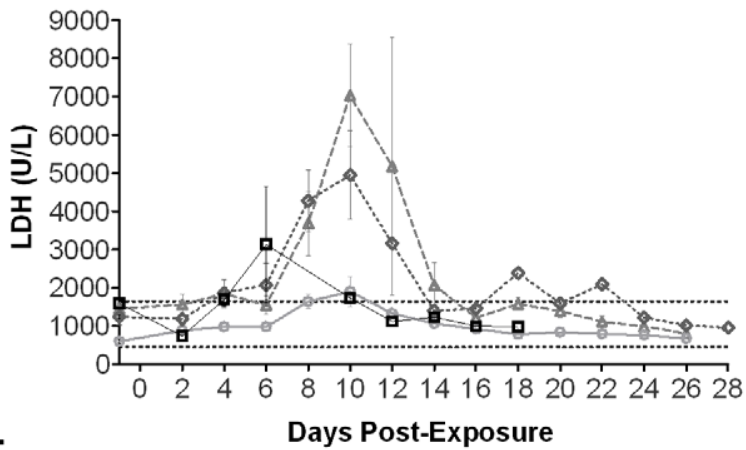

E
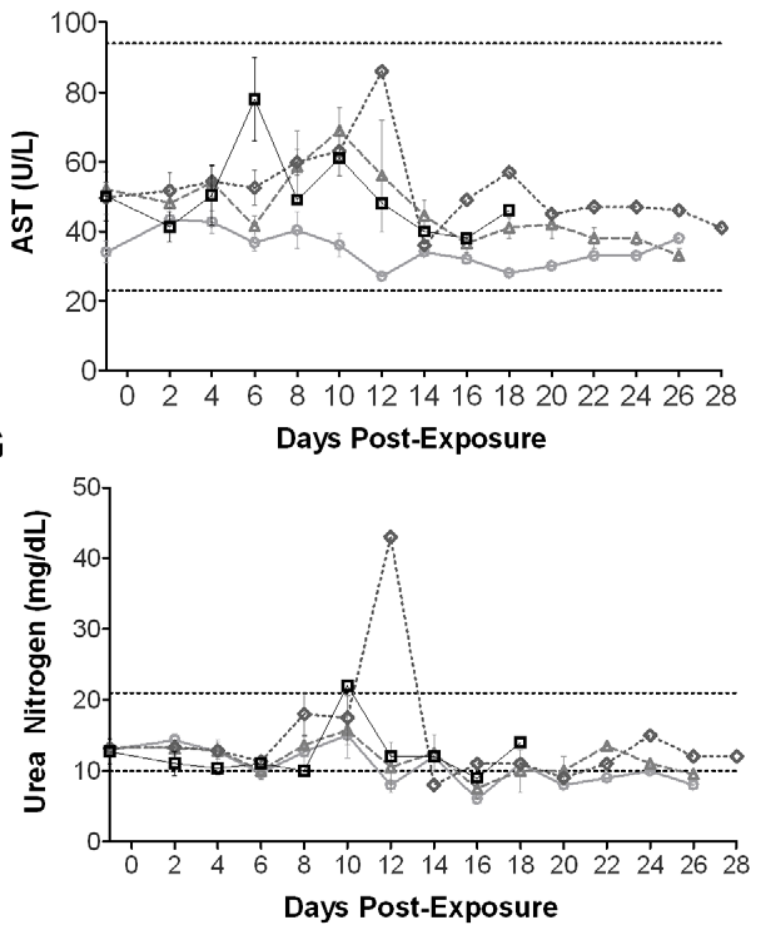

B
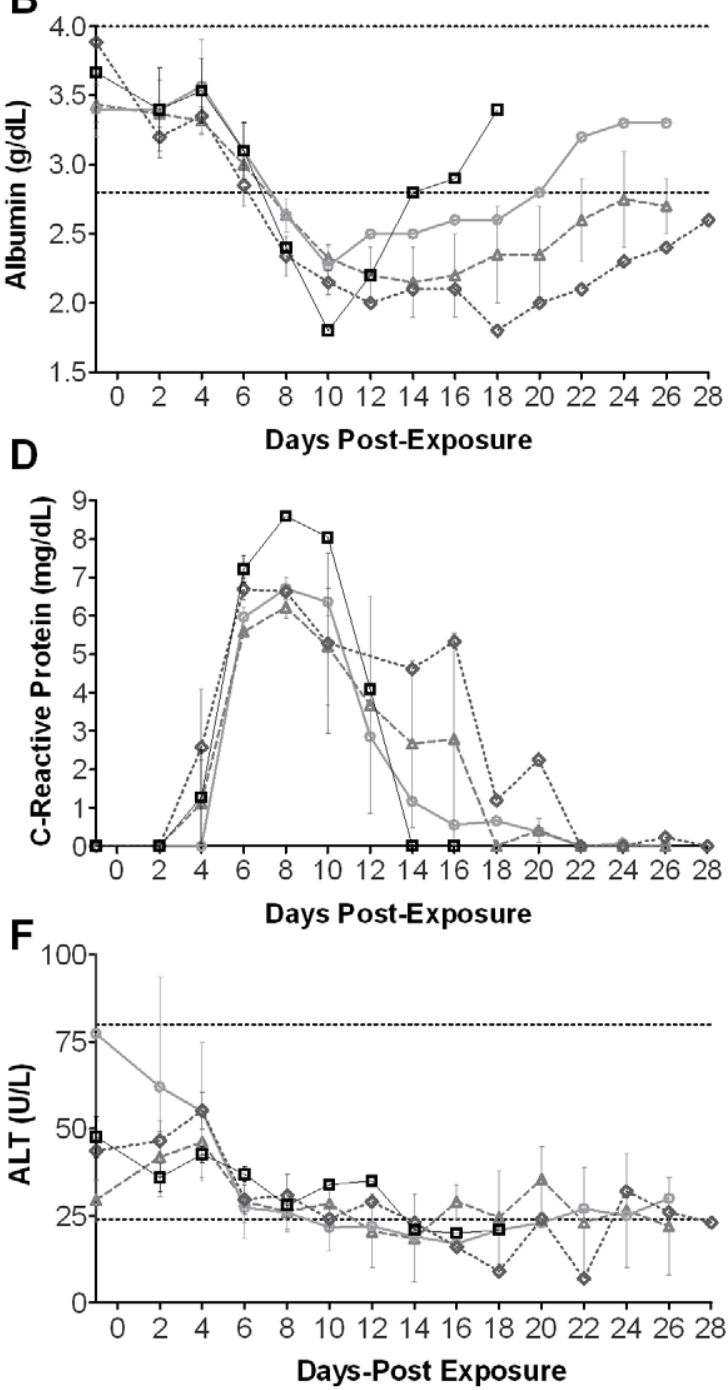

Figure 3. Serum chemistries in macaques exposed to aerosolized MPXV. The dotted lines indicate the normal reference range; n: number of animals. Graphs show average A) total protein, B) albumin, C) lactate dehydrogenase (LDH), D) C-reactive protein, E) aspartate transaminase (AST), F) and alanine transaminase (ALT), G) urea nitrogen.

doi:10.1371/journal.pone.0012880.g003 
Serum chemistries were evaluated for all macaques one day before exposure and every other day on days 2-28. Considerable changes were observed in total protein, albumin, lactate dehydrogenase $(\mathrm{LDH})$, and C-reactive protein values in all macaques over time, but the changes were similar for all groups on days -1 to 10 for total protein, albumin, and C-reactive protein (Fig. 3A-D). There were also no significant differences in serum chemistries on days $-1-10$ between survivors and nonsurvivors (data not shown). In contrast, there were significant differences in LDH levels between groups after MPXV infection $(P=0.0002)$. Alanine and aspartate amino transaminases (ALT and AST) stayed within normal ranges and groups did not differ statistically (Fig. 3E-F). Levels of blood urea nitrogen (BUN) did not differ significantly among groups (Fig. 3G). However, compared to day -1 , BUN was significantly lower for all animals on day $6(P=0.0003)$, and higher for animals surviving until day $10(P=0.0091)$.

\section{Viral load}

Viral loads in whole blood and throat swab samples from exposed macaques were assessed using real-time PCR (Figure 4AB). Viral genomes in both whole blood and throat swabs were detected as early as day 4 post-exposure. The viral load peaked on day 10 for both blood and throat swab samples and gradually decreased thereafter. There were no group-wise differences between blood or throat swab viral load on days 1-10, but the viral levels in whole blood and throat swabs were higher in nonsurvivors than survivors (blood $P=0.0445$, throat swabs $P<0.0001)$.

DNA was isolated for real-time PCR from selected tissues of macaques euthanized during acute disease and in those that were euthanized at the end of the study. As expected, lung tissue and pock lesions had the highest viral load for most exposure groups, reaching $10^{10}$ genomes/g (Fig. 5A). Spleen, gonads, axillary lymph nodes, and inguinal lymph nodes also showed high viral loads in all groups. Viral loads were much higher in the tissues from macaques euthanized during acute illness, in contrast to those that were convalescing (Fig. 5B). Virus persisted in gonads and kidneys but was reduced in other organs of survivors 28 days postexposure.

\section{Pathology}

Gross and histopathologic features of animals euthanized during acute disease (days 8-11 post-exposure) were similar to those previously reported for aerosolized MPXV [25]. Key histopathologic findings are presented in Table 2. The cause of death was attributed to primary fibrinonecrotic bronchopneumonia. Necrotizing lesions were also present in the skin, gastrointestinal tract (esophagus, stomach, duodenum, colon), lymphoid organs (tonsil, spleen, thymus, gut-associated lymphoid tissue, lymph nodes),
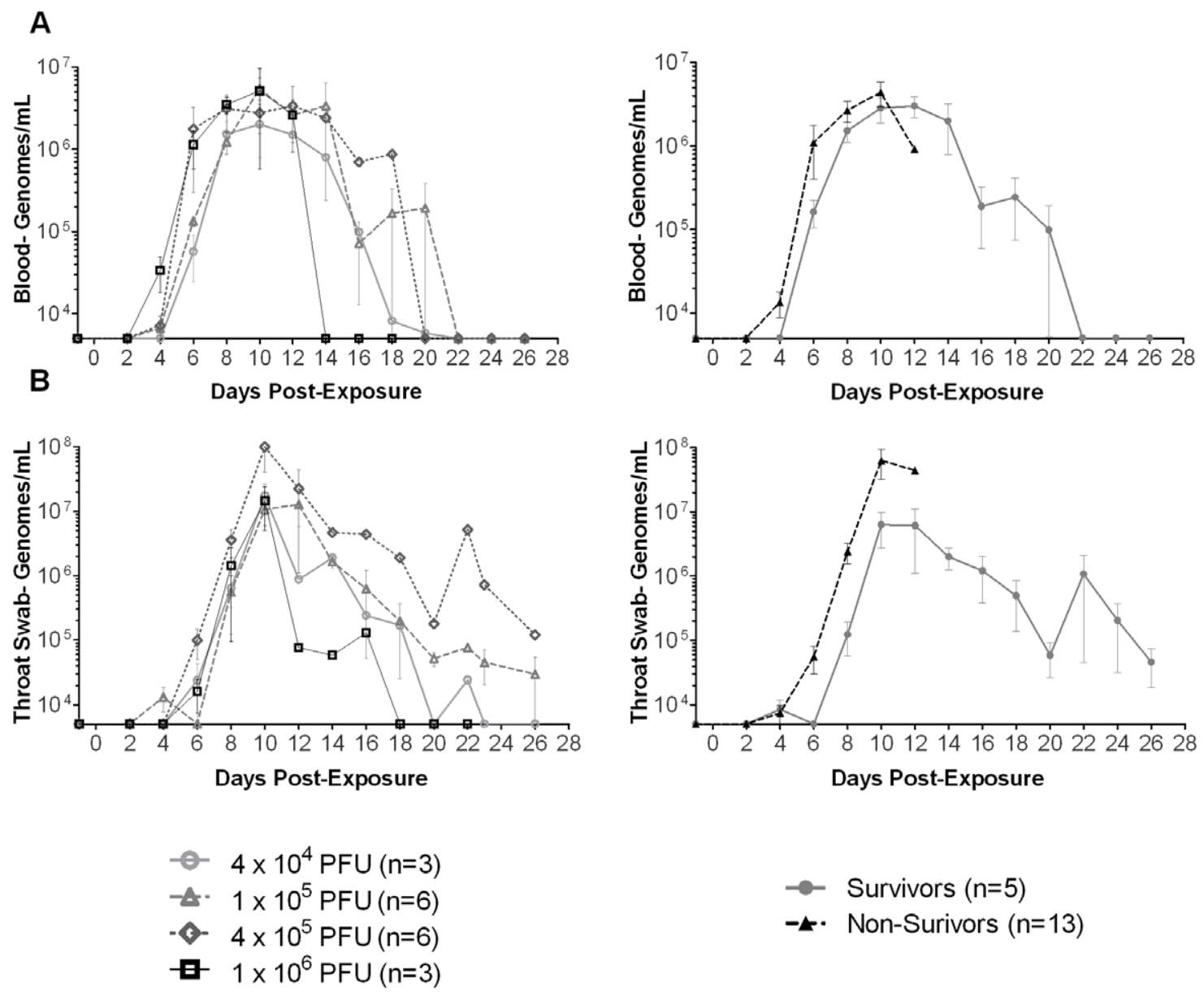

Figure 4. Average number of MPXV viral genomes in whole blood and throat swabs. The average number of MPXV viral genomes in A) whole blood, and B) throat swabs from different MPXV dosage groups, and survivors versus non-survivors (right). The $\mathrm{X}$-axis is at the limit of detection $=5000$ genomes $/ \mathrm{ml} ; \mathrm{n}$ : number of animals. doi:10.1371/journal.pone.0012880.g004 

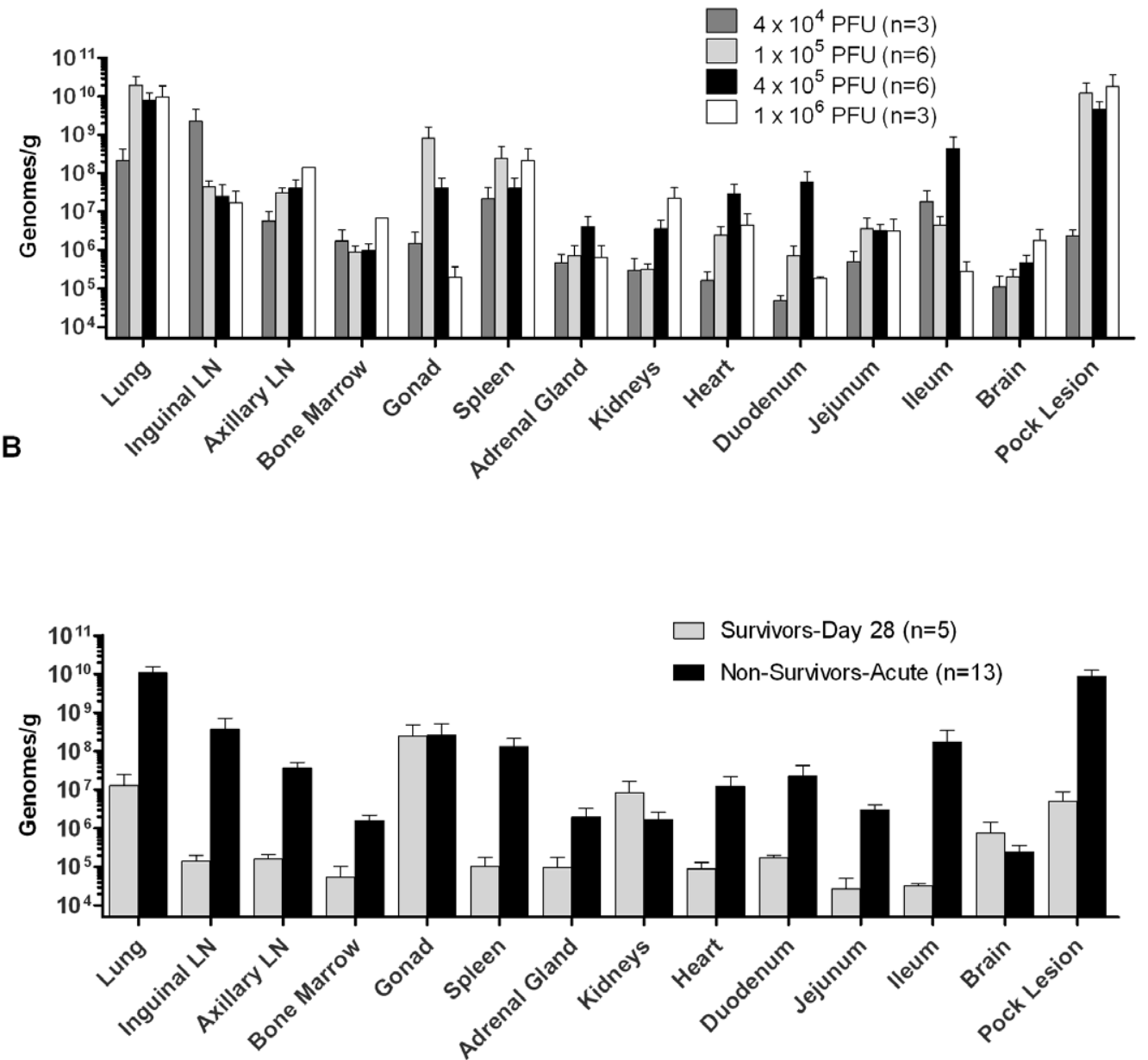

Figure 5. Viral load in tissues. A) Tissue viral load of macaques exposed to different doses of aerosolized MXPV and euthanized during the acute phase of disease (survivors are not included). B) Tissue viral load in survivors and non-survivors. The $X$-axis is at the limit of detection $=5000$ genomes $/ \mathrm{g} ; \mathrm{n}$ : number of animals. doi:10.1371/journal.pone.0012880.g005

mucosal surfaces (oral cavity, trachea, larynx), and gonads [25]. Additional necrotizing lesions were observed rarely in the prostate gland, uterus, skeletal muscle, urinary bladder, bone marrow, and conjunctiva. MPXV-associated lesions were not observed in the liver. There were mild variations in severity of lesions among the different groups, although these differences were not dosedependent.

Survivors had a variety of chronic lesions, including discrete, nodular to coalescing areas of necrosis and inflammation in the lung and mediastinal lymph nodes, chronic inflammation centered on bronchi and vessels, type II pneumocyte hyperplasia, pleural and interstitial fibrosis, and fibrous pleural adhesions. Other lesions included lymphoid hyperplasia and plasmacytosis, and chronic periadnexal and perivascular dermatitis.

Positive orthopoxvirus immunoreactivity was associated with necrotizing lesions in animals that died during acute disease. In the lungs, this was predominantly concentrated around bronchi and bronchioles (Fig. 6A-D). No poxvirus immunoreactivity was observed in normal uninfected NHP splenic tissue which served as a negative control (data not shown). The percentage of orthopoxviral antigen in lung sections of non-survivors increased with increasing dosage of virus, but the differences were not significant (Fig. 6E). In animals that survived, non cell-associated poxvirus antigen was detected in the center of discrete areas of necrosis in the lung (3/5 animals) and mediastinal lymph node (1/ 5 animals) - these findings were not dose-dependent.

\section{Discussion}

Different routes of poxvirus exposure can lead to variations in disease course in both humans and monkeys [31,32]. Many studies used i.v. delivery of MPXV as a model for smallpox and to test vaccines and countermeasures [33]. However, aerosol delivery of MPXV most closely mimics the route of natural transmission of smallpox among humans, which is by the respiratory route [22]. The pathogenesis of aerosol MPXV infection is comparable to smallpox because the infection is initiated in the respiratory mucosa followed by spread to local lymph nodes before primary 
Table 2. Key histopathologic lesions in cynomolgus macaques exposed to aerosolized MPXV.

\begin{tabular}{|c|c|c|c|c|c|c|c|c|}
\hline \multirow{2}{*}{$\begin{array}{l}\text { Tissue } \\
\text { Histopathologic Findings }\end{array}$} & \multicolumn{2}{|c|}{$4 \times 10^{4} \mathrm{PFU}$} & \multicolumn{2}{|c|}{$1 \times 10^{5} \mathrm{PFU}$} & \multicolumn{2}{|c|}{$4 \times 10^{5}$ PFU } & \multicolumn{2}{|c|}{$1 \times 10^{6}$ PFU } \\
\hline & $\mathbf{n}$ & $\%$ & $\mathbf{n}$ & $\%$ & $\mathbf{n}$ & $\%$ & $\mathbf{n}$ & $\%$ \\
\hline \multicolumn{9}{|l|}{$\begin{array}{l}\text { lung } \\
\text { bronchopneumonia, }\end{array}$} \\
\hline fibrinonecrotic & $2 / 3$ & 66 & $4 / 6$ & 66 & $5 / 6$ & 83 & $2 / 3$ & 66 \\
\hline pleuritis, necrotizing & $3 / 3$ & 100 & $4 / 6$ & 66 & $3 / 6$ & 50 & $2 / 3$ & 66 \\
\hline \multicolumn{9}{|l|}{ discrete foci of necrosis } \\
\hline /inflammation * & $1 / 3$ & 33 & 0 & 0 & $1 / 6$ & 16 & 0 & 0 \\
\hline \multicolumn{9}{|l|}{ perivascular/peribronchial } \\
\hline inflammation* & 0 & 0 & $2 / 6$ & 33 & $1 / 6$ & 16 & $1 / 3$ & 33 \\
\hline \multicolumn{9}{|l|}{ spleen } \\
\hline splenitis, necrotizing & $1 / 3$ & 33 & $2 / 6$ & 33 & $4 / 6$ & 66 & $2 / 3$ & 66 \\
\hline lymphoid depletion & $2 / 3$ & 66 & $3 / 6$ & 50 & $3 / 6$ & 50 & $2 / 3$ & 66 \\
\hline lymphoid hyperplasia** & 0 & 0 & 0 & 0 & 0 & 0 & $1 / 3$ & 33 \\
\hline \multicolumn{9}{|l|}{ mandibular lymph node } \\
\hline lymphadenitis, necrotizing & $1 / 3$ & 33 & $2 / 6$ & 33 & $5 / 6$ & 83 & $2 / 3$ & 66 \\
\hline lymphoid depletion & 0 & 0 & $2 / 6$ & 33 & $3 / 6$ & 50 & $1 / 3$ & 33 \\
\hline lymphoid hyperplasia** & $1 / 3$ & 33 & $2 / 6$ & 33 & $2 / 6$ & 33 & $1 / 3$ & 33 \\
\hline \multicolumn{9}{|l|}{ mesenteric lymph node } \\
\hline lymphadenitis, necrotizing & 0 & 0 & 0 & 0 & 0 & 0 & $1 / 3$ & 33 \\
\hline lymphoid depletion & 0 & 0 & 0 & 0 & $1 / 6$ & 16 & $2 / 3$ & 66 \\
\hline lymphoid hyperplasia** & 0 & 0 & $2 / 6$ & 33 & $1 / 6$ & 16 & 0 & 0 \\
\hline \multicolumn{9}{|l|}{ axillary lymph node } \\
\hline lymphadenitis, necrotizing & 0 & 0 & $3 / 6$ & 50 & $3 / 6$ & 50 & $2 / 3$ & 66 \\
\hline lymphoid depletion & 0 & 0 & $2 / 6$ & 33 & 0 & 0 & $2 / 3$ & 66 \\
\hline lymphoid hyperplasia** & $1 / 3$ & 33 & $2 / 6$ & 33 & $2 / 6$ & 33 & $1 / 3$ & 33 \\
\hline \multicolumn{9}{|l|}{ inguinal lymph node } \\
\hline lymphadenitis, necrotizing & 0 & 0 & $1 / 6$ & 16 & $2 / 6$ & 33 & $2 / 3$ & 66 \\
\hline lymphoid depletion & 0 & 0 & 0 & 0 & 0 & 0 & $2 / 3$ & 66 \\
\hline lymphoid hyperplasia* & $1 / 3$ & 33 & $2 / 6$ & 33 & $2 / 6$ & 33 & $1 / 3$ & 33 \\
\hline \multicolumn{9}{|c|}{ tracheobronchial lymph node } \\
\hline lymphadenitis, necrotizing & $2 / 3$ & 66 & $2 / 6$ & 33 & $4 / 6$ & 66 & $1 / 2$ & 50 \\
\hline lymphoid depletion & $2 / 3$ & 66 & $3 / 6$ & 50 & $3 / 6$ & 50 & 0 & 0 \\
\hline lymphoid hyperplasia* & 0 & 0 & $1 / 6$ & 16 & $1 / 6$ & 16 & $1 / 2$ & 50 \\
\hline \multicolumn{9}{|l|}{ discrete foci of necrosis } \\
\hline /inflammation * & 0 & 0 & 0 & 0 & 0 & 0 & $1 / 2$ & 50 \\
\hline
\end{tabular}

* = lesion seen in survivors.

doi:10.1371/journal.pone.0012880.t002

viremia ensues. A major pitfall of the i.v. MPXV infection model is that the initial infection of respiratory tissue, incubation, and prodromal phases are bypassed with the direct initiation of viremia. The same phenomenon has occurred in human MPXV infections initiated by scratch or bite versus those presumed to have occurred by respiratory exposure [31]. This is an important caveat when the utility of these models is meant to test possible vaccines and treatments in which the efficacy may depend on protecting the respiratory mucosa and targeting subsequent early stages of the infection, which are not represented in the i.v. challenge model.

A marmoset (Callithrix jacchus) model of intranasal calpox, a strain of cowpox virus, was recently evaluated [14]. Advantages of this model include the lower lethal dose required and the relative ease of husbandry of marmosets compared to larger species of NHPs. However, there are several disadvantages to the intranasal marmoset calpox model. First, the intranasal route is less physiologically relevant to a naturally occurring poxvirus infection, despite the relative technical ease at which intranasal challenge can be accomplished compared to aerosol challenge. Additionally, the clinical disease course was less similar to that of MPXV or VARV infection of humans, due to the appearance of very few pox lesions and the appearance of observable clinical disease of short duration just prior to death. Finally, perhaps because of the small size of the marmosets, blood was not drawn with great frequency and serum chemistries and CBCs were not performed, thus leaving many features of the clinical disease course unexplored. 


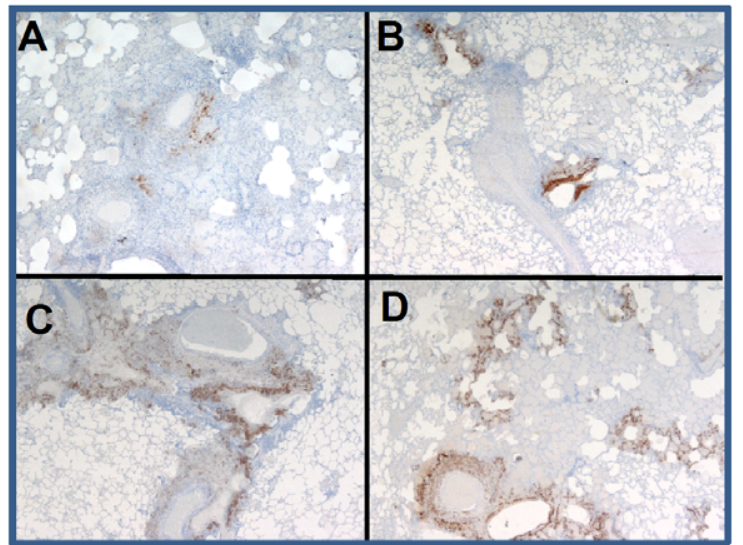

\section{E}

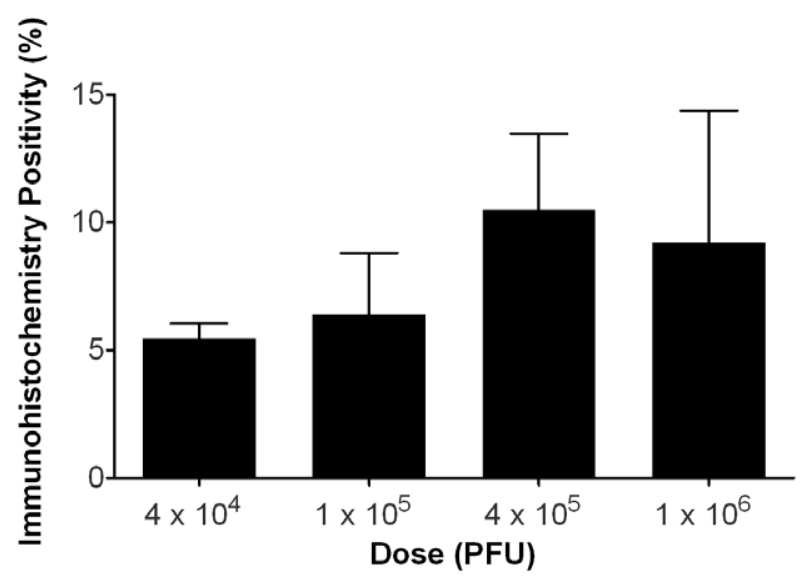

Figure 6. Pathology and presence of MPXV antigen in lung tissue. Figures A-D are histological sections of lung tissues from cynomolgus macaques infected via aerosolized MPXV. Positive immunoreactivity for orthopoxvirus antigen, shown as brown staining, is associated with necrotizing lesions primarily concentrated around bronchi and bronchioles. [Immunoperoxidase method using rabbit polyclonal antibody to vaccinia virus; original magnification $\times 40$ (Figure $6 \mathrm{~A}$ ) or $\times 20$ (Figure $6 \mathrm{~B}, \mathrm{C}, \mathrm{D})$ ]. A) $4 \times 10^{4}$ PFU (day 10 postexposure). B) $1 \times 10^{5} \mathrm{PFU}$ (day 8 post-exposure). C) $4 \times 10^{5}$ PFU (day 11 post-exposure). D) $1 \times 10^{6} \mathrm{PFU}$ (day 9 post-exposure). E) Percent immunoreactivity in the lungs of non-survivors by dosage group, measured by digital microscopy image analysis.

doi:10.1371/journal.pone.0012880.g006

An intratracheal infection model deposits virus directly into airways but without regard to particle size and the physiological deposition that occurs during the process of inhalation. Fibrinonecrotic bronchopneumonia was described in animals that received $10^{7} \mathrm{pfu}$ MPXV by i.t. inoculation, as was also the case in animals infected by the aerosol route in this study as well as the study by Zaucha and colleagues [25,34]. Intratracheal MPXV infection with a comparable dose of virus to that used in this study $\left(10^{6} \mathrm{PFU}\right)$ resulted in a strikingly similar curve for viremia to that seen in this study, with a large peak followed by a smaller peak at approximately 22 days post exposure [34]. However, the timing of the first peak was delayed by 5 days in i.t. exposed macaques compared to aerosol infection, and the amount of virus detected by qPCR was approximately 100 -fold lower. This suggests that local replication is more pronounced after aerosol delivery compared to the i.t. route. Taken together, i.t. inoculation is therefore not a substitute for the aerosol route of infection.
Human MPXV infection has a mortality ranging from $1.5 \%$ to $10 \%$ according to epidemiologic data, and is clinically indistinguishable from human smallpox except for a greater frequency of lymphadenopathy $[3,35,36]$. Likewise, a 1961 study reported similar diseases after aerosol infection of cynomolgus macaques with MPXV compared to several VARV strains [37]. While MPXV is thought to have a lower potential for human-to-human transmission than VARV, it is currently the most troublesome orthopoxvirus for humans, with sporadic outbreaks occurring most commonly in the African Congo [35]. An outbreak of human monkeypox occurred in the Midwestern US in 2003 after contact with prairie dogs infected with a West African strain of MPXV [38]. MPXV-infected humans had a longer incubation and shorter fever duration than the macaques in this study, which may be due in part to a presumed lower dose of a natural environmental exposure or differences in virulence between the West African strain causing the US outbreak and the Central African strain used for this study [17,39].

Hematology data indicated that WBC levels were significantly increased from the baseline levels by day 4 post-exposure $(P=0.0013)$, with the WBC levels in some dosage groups increasing above the normal range by day 10 . The increased neutrophils and decreased lymphocytes - although not true neutrophilia and lymphopenia, since the values were within reference ranges - would be consistent with an inflammatory leukogram. Similarly, leukocytosis was observed in $45 \%$ of human monkeypox cases [38]. Thrombocytopenia was seen in $35 \%$ of human patients and significant decreases in platelet levels also occurred on day 6 post-exposure in macaques. Histologic evaluation of bone marrow did not reveal significant pathology; thus, the mechanism of decreased platelets was unlikely due to decreased production but more likely due to increased consumption or removal due to viral infection.

In contrast to the human cases that had low BUN levels (61\%), MPXV exposed macaques had normal BUN indicating that decreased production of urea or reduced availability of ammonia for urea synthesis tends to occur in humans but may not occur in MPXV infected macaques [38]. The animals in this study had no pathologic abnormalities in the kidneys despite the persistence of virus in the kidneys of animals surviving infection. Macaques in the $1 \times 10^{5} \mathrm{PFU}, 4 \times 10^{5} \mathrm{PFU}$, and $1 \times 10^{6} \mathrm{PFU}$ groups had significantly elevated AST at day 10 post-exposure compared to day -1 $(P=0.002)$. Likewise, high transaminase levels were detected in $50 \%$ of MPXV infected humans. Although AST levels in MPXV infected macaques were within the normal range, the levels were elevated compared to baseline levels.

$\mathrm{LDH}$ is a protein that aids in the removal of lactate from tissues. Lactate is the end product when anaerobic glycolysis occurs in low oxygen conditions. Increased LDH in MPX infection indicates increased lactate and therefore decreased oxygen in the body. Since aerosolized MPX infection affects the lungs, this is a possible cause of elevated LDH levels. Additionally, because LDH lacks tissue specificity and the major sources of high serum LDH activity include muscle, liver, and erythrocytes, we cannot rule out these sources as contributing factors. As observed in 50\% of MPXV infected humans diagnosed with hypoalbuminemia, significant decreases in albumin levels were also seen in this study in each of the dosage groups by day 6 post-exposure $(P=0.0056)$. Hypoproteinemia and hypoalbuminemia likely resulted from anorexia which may have been the consequence of lesions within the mouth and esophagus. Thus, there are a number of similarities between monkeypox disease in humans and aerosol exposed macaques.

The pathologic findings in this study are similar to those reported by Zaucha et al. [25]. In animals with acute disease, fibrinonecrotic bronchopneumonia was the most distinctive lesion 
observed. In animals surviving the infection, nodular to coalescing foci of necrosis and inflammation appeared to be centered on large airways. Other lesions in animals with acute disease included necrotizing lesions in the skin, mucosal surfaces, lymphoid tissues, and gonads. Cutaneous lesions ranged from very few in some animals to too numerous to count in others. Additional lesions seen in this study, albeit infrequently, were necrotizing lesions in the prostate gland, uterus, skeletal muscle, and urinary bladder. Hepatic lesions were not observed in our study, whereas Zaucha et al. described disseminated hepatitis in $13 \%$ of MPXV infected animals.

Generally, a dose of $10^{6} \mathrm{pfu} \mathrm{MPXV}$ given by aerosol to cynomolgus macaques is uniformly lethal and would be an appropriate dose for testing of orthopoxvirus countermeasures (data not shown). However, in this report, one of three animals survived challenge at this dose, possibly due to individual differences in the host immune response. Future studies should be done to determine which primate host responses are amenable to survival following aerosol exposure of MPXV.

Interestingly, the average weight of the survivors was $20 \%$ heavier than that of the non-survivors. Macaques were between 4 and 6 years old, therefore age differences were not likely a factor in survival. Of the five animals that survived, four were male, indicating that males, which tend to be heavier, may be more resistant to MPXV. Increased survival in male macaques could be due either to gender or heavier weight. Among humans, correlations of MPXV disease severity with weight, gender, or age have not been made, although pediatric patients were more likely than adults to be admitted into an intensive care unit in the 2003 US outbreak [38].

Unlike other animal models of orthopoxvirus infection, the model presented here utilizes the respiratory route which is the

\section{References}

1. Shchelkunov SN, Totmenin AV, Babkin IV, Safronov PF, Ryazankina OI, et al. (2001) Human monkeypox and smallpox viruses: genomic comparison. FEBS Lett 509: 66-70.

2. Parker S, Nuara A, Buller RM, Schultz DA (2007) Human monkeypox: an emerging zoonotic disease. Future Microbiol 2: 17-34.

3. Nalca A, Rimoin AW, Bavari S, Whitehouse CA (2005) Reemergence of monkeypox: prevalence, diagnostics, and countermeasures. Clin Infect Dis 41: $1765-1771$.

4. Jezek Z, Fenner F (1988) Human monkeypox. Basel; New York: Karger.

5. Fine PE, Jezek Z, Grab B, Dixon H (1988) The transmission potential of monkeypox virus in human populations. Int J Epidemiol 17: 643-650.

6. Marriott KA, Parkinson CV, Morefield SI, Davenport R, Nichols R, et al. (2008) Clonal vaccinia virus grown in cell culture fully protects monkeys from lethal monkeypox challenge. Vaccine 26: 581-588.

7. Earl PL, Americo JL, Wyatt LS, Eller LA, Whitbeck JC, et al. (2004) Immunogenicity of a highly attenuated MVA smallpox vaccine and protection against monkeypox. Nature 428: 182-185.

8. Earl PL, Americo JL, Wyatt LS, Espenshade O, Bassler J, et al. (2008) Rapid protection in a monkeypox model by a single injection of a replication-deficient vaccinia virus. Proc Natl Acad Sci U S A 105: 10889-10894.

9. Edghill-Smith Y, Golding H, Manischewitz J, King LR, Scott D, et al. (2005) Smallpox vaccine-induced antibodies are necessary and sufficient for protection against monkeypox virus. Nat Med 11: 740-747.

10. Handley L, Buller RM, Frey SE, Bellone C, Parker S (2009) The new ACAM2000 vaccine and other therapies to control orthopoxvirus outbreaks and bioterror attacks. Expert Rev Vaccines 8: 841-850.

11. Nigam P, Earl PL, Americo JL, Sharma S, Wyatt LS, et al. (2007) DNA/MVA HIV-1/AIDS vaccine elicits long-lived vaccinia virus-specific immunity and confers protection against a lethal monkeypox challenge. Virology 366: 73-83.

12. Garza NL, Hatkin JM, Livingston V, Nichols DK, Chaplin PJ, et al. (2009) Evaluation of the efficacy of modified vaccinia Ankara (MVA)/IMVAMUNE against aerosolized rabbitpox virus in a rabbit model. Vaccine 27: 5496-5504.

13. Food and Drug Administration H (2002) New Drug and Biological Drug Products; Evidence Needed to Demonstrate Effectiveness of New Drugs When Human Efficacy Studies Are Not Ethical or Feasible. 21 CFR Parts 314 and 601.

14. Kramski M, Matz-Rensing K, Stahl-Hennig C, Kaup FJ, Nitsche A, et al. A novel highly reproducible and lethal nonhuman primate model for orthopox virus infection. PLoS One 5: e10412. natural route of transmission for human VARV infections and a secondary route for human MPXV infections. We have demonstrated that aerosol infection of cynomolgus macaques with MPXV has a number of parallels to human monkeypox and smallpox diseases. Therefore, an aerosol infection model in NHPs is important for understanding orthopoxvirus pathogenesis as well as for future evaluation of novel vaccine and therapeutic candidates.

\section{Acknowledgments}

The authors thank the personnel in the Aerosol Services Branch of the Center for Aerobiological Sciences for conducting the aerosol sprays of animals, the personnel of the Veterinary Medicine Division for the care and handling of the animals, and Larry Sullivan from the Clinical Laboratory for processing samples in these studies. Furthermore, the authors gratefully acknowledge Gale Krietz, Neil Davis, and Jeff Brubaker for histology preparations, Chris Mech for immunohistochemistry, Diana Fisher for her assistance with statistics, and Katheryn Kenyon for editorial revision. We also thank Robert Johnson and Blair Osborn (NIAID) for excellent discussions during the study and also for critically reviewing the manuscript.

Disclaimer: Opinions, interpretations, conclusions, and recommendations are those of the authors and are not necessarily endorsed by the U.S. Army or the Department of Defense.

\section{Author Contributions}

Conceived and designed the experiments: AN. Performed the experiments: AN VAL NLG OMF JLC JMH. Analyzed the data: AN EEZ OMF JLC JMH. Contributed reagents/materials/analysis tools: AN VAL NLG EEZ JLG JMH. Wrote the paper: AN EEZ JLC JMH.

15. Adams MM, Rice AD, Moyer RW (2007) Rabbitpox virus and vaccinia virus infection of rabbits as a model for human smallpox. J Virol 81: 11084-11095.

16. Huggins J, Goff A, Hensley L, Mucker E, Shamblin J, et al. (2009) Nonhuman primates are protected from smallpox virus or monkeypox virus challenges by the antiviral drug ST-246. Antimicrob Agents Chemother 53: 2620-2625.

17. Hutson CL, Olson VA, Carroll DS, Abel JA, Hughes CM, et al. (2009) A prairie dog animal model of systemic orthopoxvirus disease using West African and Congo Basin strains of monkeypox virus. J Gen Virol 90: 323-333.

18. Schriewer J, Buller RM, Owens G (2004) Mouse models for studying orthopoxvirus respiratory infections. Methods Mol Biol 269: 289-308.

19. Smee DF (2008) Progress in the discovery of compounds inhibiting orthopoxviruses in animal models. Antivir Chem Chemother 19: 115-124.

20. Stittelaar KJ, Neyts J, Naesens L, van Amerongen G, van Lavieren RF, et al. (2006) Antiviral treatment is more effective than smallpox vaccination upon lethal monkeypox virus infection. Nature 439: 745-748.

21. Tesh RB, Watts DM, Sbrana E, Siirin M, Popov VL, et al. (2004) Experimental infection of ground squirrels (Spermophilus tridecemlineatus) with monkeypox virus. Emerg Infect Dis 10: 1563-1567.

22. Henderson DA (2009) Smallpox: the death of a disease: the inside story of eradicating a worldwide killer. Amherst, N.Y.: Prometheus Books.

23. Swearengen JR (2006) Biodefense: research methodology and animal models. Boca Raton: CRC Taylor \& Francis.

24. Jahrling PB, Hensley LE, Martinez MJ, Leduc JW, Rubins KH, et al. (2004) Exploring the potential of variola virus infection of cynomolgus macaques as a model for human smallpox. Proc Natl Acad Sci U S A 101: 15196-15200.

25. Zaucha GM, Jahrling PB, Geisbert TW, Swearengen JR, Hensley L (2001) The pathology of experimental aerosolized monkeypox virus infection in cynomolgus monkeys (Macaca fascicularis). Lab Invest 81: 1581-1600.

26. Besch TK, Ruble DL, Gibbs PH, Pitt ML (1996) Steady-state minute volume determination by body-only plethysmography in juvenile rhesus monkeys. Lab Anim Sci 46: 539-544.

27. Hartings JM, Roy CJ (2004) The automated bioaerosol exposure system: preclinical platform development and a respiratory dosimetry application with nonhuman primates. J Pharmacol Toxicol Methods 49: 39-55.

28. Nalca A, Hatkin JM, Garza NL, Nichols DK, Norris SW, et al. (2008) Evaluation of orally delivered ST-246 as postexposure prophylactic and antiviral therapeutic in an aerosolized rabbitpox rabbit model. Antiviral Res 79: 121-127.

29. Prophet EB, Mills B, Arrington JB, Sobin LH (1992) Laboratory Methods for Histotechnology. Washington, D.C.: Armed Forces Institute of Pathology. 
30. Bauer KD, de la Torre-Bueno J, Diel IJ, Hawes D, Decker WJ, et al. (2000) Reliable and sensitive analysis of occult bone marrow metastases using automated cellular imaging. Clin Cancer Res 6: 3552-3559.

31. Reynolds MG, Yorita KL, Kuehnert MJ, Davidson WB, Huhn GD, et al. (2006) Clinical manifestations of human monkeypox influenced by route of infection. J Infect Dis 194: 773-780.

32. Hahon N (1961) Smallpox and related poxvirus infections in the simian host. Bacteriol Rev 25: 459-476.

33. Jordan R, Goff A, Frimm A, Corrado ML, Hensley LE, et al. (2009) ST-246 antiviral efficacy in a nonhuman primate monkeypox model: determination of the minimal effective dose and human dose justification. Antimicrob Agents Chemother 53: 1817-1822.

34. Stittelaar KJ, van Amerongen G, Kondova I, Kuiken T, van Lavieren RF, et al. (2005) Modified vaccinia virus Ankara protects macaques against respiratory challenge with monkeypox virus. J Virol 79: 7845-7851.
35. Rimoin AW, Kisalu N, Kebela-Ilunga B, Mukaba T, Wright LL, et al. (2007) Endemic human monkeypox, Democratic Republic of Congo, 2001-2004. Emerg Infect Dis 13: 934-937.

36. Hutin YJ, Williams RJ, Malfait P, Pebody R, Loparev VN, et al. (2001) Outbreak of human monkeypox, Democratic Republic of Congo, 1996 to 1997. Emerg Infect Dis 7: 434-438.

37. Hahon N, Mc GM (1961) Air-borne infectivity of the variola-vaccinia group of poxviruses for the cynomolgus monkey, Macaca irus. J Infect Dis 109: 294-298.

38. Huhn GD, Bauer AM, Yorita K, Graham MB, Sejvar J, et al. (2005) Clinical characteristics of human monkeypox, and risk factors for severe disease. Clin Infect Dis 41: 1742-1751.

39. Hutson CL, Abel JA, Carroll DS, Olson VA, Braden ZH, et al. Comparison of West African and Congo Basin monkeypox viruses in BALB/c and C57BL/6 mice. PLoS One 5: e8912. 